\title{
Brain studies of mouse models for neurogenetic disorders using in vivo magnetic resonance imaging (MRI)
}

\author{
R Frank Kooy ${ }^{\star 1}$, Marleen Verhoye ${ }^{2}$, Vance Lemmon ${ }^{3}$ and Annemie Van Der Linden ${ }^{2}$ \\ ${ }^{1}$ Department of Medical Genetics, University of Antwerp, Antwerp, Belgium; ${ }^{2}$ Bio-Imaging Laboratory, University of \\ Antwerp, Antwerp, Belgium; ${ }^{3}$ Department of Neurosciences, Case Western Reserve University, Cleveland, Ohio, \\ USA
}

Magnetic resonance imaging (MRI) is a technique commonly used to detect neural abnormalities in routine clinical practice. It is perhaps less well known that the technique can be adapted to measure various anatomical and physiological features of small laboratory rodents. This review focuses on the potential of the MRI technique to image the brain of (transgenic) mouse models for neurological diseases, and aims to introduce these exciting new technological developments to the non-specialist reader. European Journal of Human Genetics (2001) 9, $153-159$.

Keywords: magnetic resonance imaging; fragile $\mathrm{X}$ syndrome; $\mathrm{X}$-linked hydrocephalus; knockout mouse; mental retardation; brain structure

\section{Introduction}

The recent progress in gene technology resulted in an explosive increase of genetically modified mouse models. Such models are only valuable if they demonstrate a substantial similarity with the human pathology. Since most typical neurological disorders include age-dependent neuronal and behavioural changes, it is important to accurately validate newly developed mice models for these characteristics. ${ }^{1}$ Only in vivo non-invasive methods allow the follow up of age-dependent changes and allow to combine the observed neuropathologic features with observed abnormalities in behaviour and brain function within the same mouse. In spite of the impact of in vivo imaging techniques such as magnetic resonance imaging $(\mathrm{MRI})^{2}$ in routine clinical practice and in human neuroscience (Figure 1), MRI studies on mice are scarce. The reason for the delayed exploitation of these in vivo methods for transgenic mice research is the fact that technical developments allowed adaptation of the techniques from the human clinic to a micro version suitable

*Correspondence: R Frank Kooy, Department of Medical Genetics, University of Antwerp, Universiteitsplein 1, 2610 Antwerp, Belgium. Tel: +32 3 8202630; Fax: +32 3 8202566; E-mail: fkooy@uia.ac.be Received 4 October 2000; accepted 16 November 2000 to measure small animals only recently. ${ }^{3}$ This was stimulated by the progressive interest (and expansion) in knockout and transgenic mouse models and the more general pressure to perform research in an animal saving and animal friendly environment. MRI provides an excellent method for the proposed characterisation of genetically modified mice. It is a powerful, non-invasive technique that provides high-resolution brain images. It can be performed on anesthetized animals, so that the animal does not have to be sacrificed and can be measured over a series of time intervals. The latter allows comparison of mouse data with patient MRI data base material.

This review introduces magnetic resonance imaging as a tool to study the brain of mouse models with neurogenetic diseases and presents an overview of initial MRI studies that have pioneered this technique to study neuroanatomic abnormalities in genetically modified mice. In addition, possible future applications are discussed.

\section{Magnetic resonance imaging}

MRI is based on the registration of the resonance energy transmitted by protons placed in a magnetic field that return to equilibrium after excitation. To start an MRI recording, the sample (eg an animal) is positioned in a strong magnetic field 


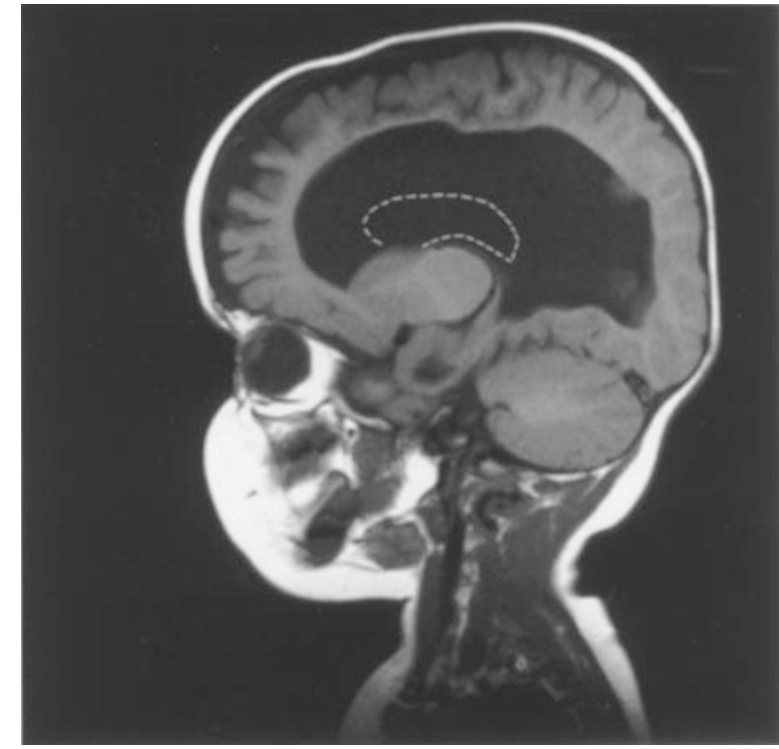

Figure 1 Sagittal MRI recording of a child affected with Xlinked hydrocephalus. Note gross enlargement of the lateral ventricles. The dotted line indicates the shape of a normal-sized ventricle. In contrast to the animal illustrations shown, the ventricle appears black due to the selection of different MRIparameters.

(Figure 2A). As hydrogen nuclei (protons) can be considered as tiny magnets, the magnetic field causes protons to orientate parallel or antiparallel with respect to the magnetic field. By subsequent application of a sequence of radio frequency pulses, administered through the radio frequency antenna (Figure 2B), the protons are forced away from their equilibrium position. When excitation is interrupted, the protons will return or 'relax' to their equilibrium position and the resulting resonance is recorded and used to generate MR images. Together with the application of magnetic field gradients to localise the concentration and relaxation properties of protons $\left({ }^{1} \mathrm{H}\right)$, primarily in water, a digital image is obtained which reflects the spatial distribution of ${ }^{1} \mathrm{H}$ in the object. By choosing an appropriate pulse sequence, the intensity of an MR image can be made to reflect one or more of several MRI parameters. Such parameters, in turn, are sensitive to the physiochemical environment of the ${ }^{1} \mathrm{H}$, eg whether the protons are found in free water, in bound water, or in $\mathrm{CH}_{2}$ groups of lipids. While proton density refers to concentration of ${ }^{1} \mathrm{H}$ nuclei, the $\mathrm{T}_{2}$ or spin-spin relaxation time depends on ratio free-to-bound water, the haemodynamics, and the oxygenation status of the tissue. The contribution of macromolecules and lipids is reflected by the $\mathrm{T}_{1}$ or spin-lattice relaxation time. The applied imaging sequences will determine whether an image will be proton density-, $\mathrm{T}_{1}$ - or $\mathrm{T}_{2}$-weighted.

MRI recordings can be acquired in any desired direction, as a series of consecutive slices (Figure 3) or as an entire 3D set.

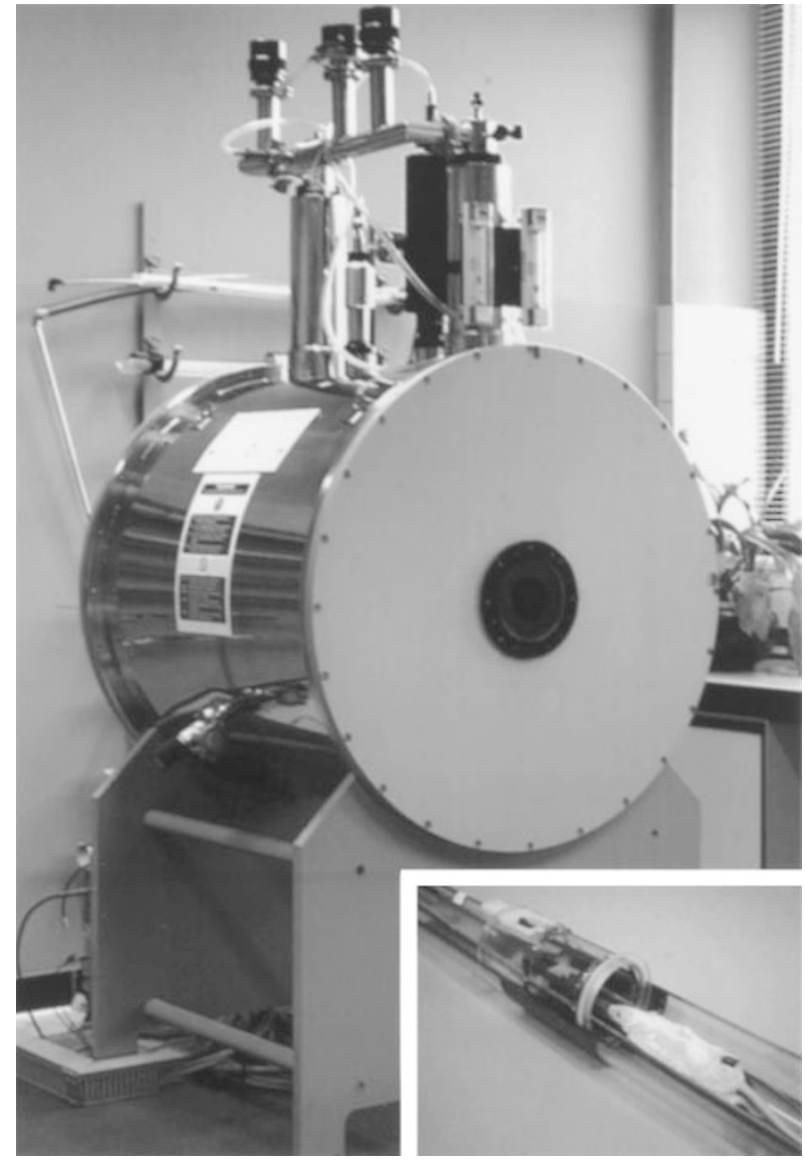

Figure 2 7T magnet with horizontal $8 \mathrm{~cm}$ diameter bore of a SMIS (UK) MR instrument providing access to an animal holding system (see insert) equipped with an RF antenna in which the mouse can be inserted.

Appropriate software allows 3D reconstruction from the obtained 3D data set (Figure 4).

\section{Why study mice?}

The mouse (and its close relative, the rat) have the most intensively studied nervous systems of all animals. This is mainly due to their relatively inexpensive costs and rapid breeding rates. Literally tens of thousands of studies have been performed on the organisation and function of their nervous systems: from studies of axonal pathways, neurotransmitter expression patterns, and synaptic organisation to physiological and behavioural experiments. Consequently, there is a wealth of information about the rodent central nervous system and its similarities and differences with primates and humans. This, combined with the detailed knowledge of mouse genetics and the relative ease with which the genome can be manipulated, makes mice the preferred system to use genetic approaches to model human diseases of the nervous system. This is not to say mice are the 
ideal model system. The small size of mice makes some types of experiments difficult.

Moreover, there is often concern expressed regarding the usefulness of the mouse nervous system as a model for the human nervous system. However, it is important to consider the alternative models. The brains of submamma-
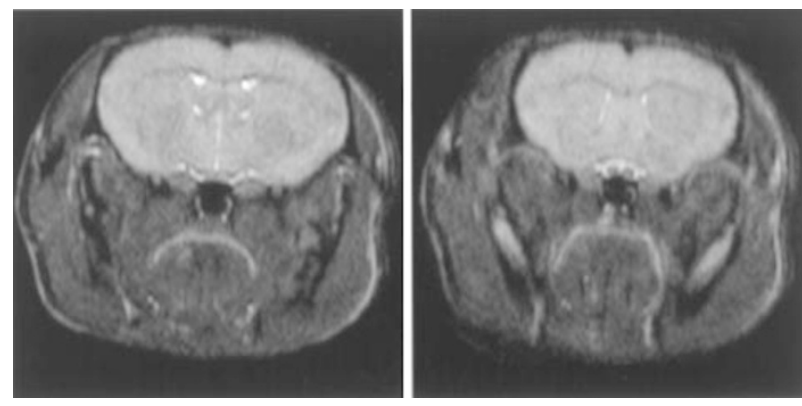

Figure 3 Two in vivo obtained coronal $\mathrm{T}_{2}$-weighted high resolution MRI images of the head of a control mouse displaying the brain and a clear distinction between cerebrospinal fluid (3rd and lateral ventricles as highlighted structures), white matter structures (eg corpus callosum as dark lines) and gray matter.

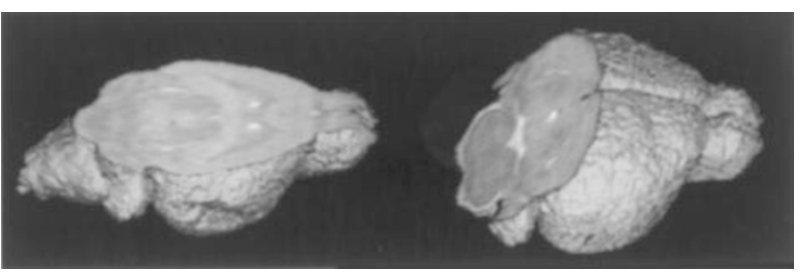

Figure 4 3D MR image of a live fragile $X$ mouse. This is the result of a set of MR images obtained from the head of the mouse and submitted to an image processing protocol resulting in the systematic segmentation of the brain and subsequent 3D reconstruction. lian models such as chick, frog, and zebra fish have brains that are substantially different from mammals. For example, the submammalian cerebellum is homologous to the mammalian vermis, while the cerebral hemispheres in mammals are not represented in these animals. It is true that mammals 'higher' than rodents have a central nervous system with organisations more similar to humans and the larger size of some of these mammals facilitates certain types of studies. However, the larger size comes with disadvantages of slower reproduction and development and lack of powerful genetics.

\section{Neuroanatomic mouse studies}

The use of MRI to study the neuroanatomy of the brain of knockout or otherwise genetically modified mouse models has been relatively limited (see Table 1). A first study that made use of MRI to determine possible brain abnormalities of a mutant mouse model appeared in $1992 .{ }^{4}$ In this study, the brain of a mouse model for Down syndrome, containing three copies of mouse chromosome 16 was compared with control littermates. Formalin-fixed animals at day 17 of gestation were imaged. Although the primary aim of this study was to detect placental abnormalities, the brain was analysed too. Under the conditions used, hardly any details were visible within the brain, but it was noticed that the trisomy 16 fetuses had a smaller cerebellum. Histological sections confirmed this observation.

The same group of authors reported near microscopic resolution of the mouse brain 2 years later. ${ }^{5}$ The brain of two control BALB/c mice were imaged in vivo at 38 days of age and compared with histological slices of littermates from the same age. Structural features of the cerebral cortex, hippocampus, thalamus, cerebellum, and brain stem could be clearly recognized on $125 \mu \mathrm{m}$ thick $\mathrm{T}_{2}$-weighted coronal slices. Within these brain structures, regions of higher signal intensity could be discerned, corresponding to perikaryal and

Table 1 Neuroanatomic differences observed with MRI between human syndromes and corresponding mouse models

\begin{tabular}{|c|c|c|c|c|}
\hline Human disease & $\begin{array}{l}\text { Principal neurologic abnormalities reported } \\
\text { in human MRI studies }\end{array}$ & Mouse model & MRI mouse brain abnormalities & Reference \\
\hline Down syndrome & & trisomie 16 & decreased cerebellar volume & 4 \\
\hline Phenylketonuria (PKU) & demyelination of deep cerebral white matter & Pah $^{\text {enu } 2}$ mutant & none & 5 \\
\hline $\begin{array}{l}\text { Aspartylglucosaminuria } \\
\text { (AGU) }\end{array}$ & $\begin{array}{l}\text { cerebral atrophy } \\
\text { hypointensity of deep gray matter }\end{array}$ & AGA mutant & $\begin{array}{l}\text { cerebral atrophy } \\
\text { hypointensity of deep gray matter }\end{array}$ & 8 \\
\hline Encephalitus & focal brain damage & virus inoculation & focal brain damage & 6 \\
\hline Scrapie & spongiform encephalopathie & $\begin{array}{l}\text { PrP }{ }^{C} \text { overexpressing } \\
\text { neural grafts }\end{array}$ & spongiform encephalopathie & 7 \\
\hline $\begin{array}{l}\text { X-linked } \\
\quad \text { hydrocephalus }\end{array}$ & $\begin{array}{l}\text { enlarged ventricular system } \\
\text { aqueduct of Sylvius stenosis } \\
\text { reduced size of cerebellar }\end{array}$ & L1cam1 knockout & $\begin{array}{l}\text { enlarged ventricular system } \\
\text { altered shape of the Sylvius } \\
\text { aqueduct } \\
\text { reduced cerebellar vermis }\end{array}$ & 11 \\
\hline Fragile $X$ syndrome & $\begin{array}{l}\text { reduced size of cerebellar vermis } \\
\text { increased volume of total brain, } \\
\text { ventricular system, hippocampus, } \\
\text { and subcortical gray }\end{array}$ & Fmr1 knockout & none & 17 \\
\hline
\end{tabular}


dendritic-rich regions. In the same study, phenylalanine hydroxylase-deficient mice, a mouse model for the human disorder phenylketonuria (PKU) were compared with control littermates. Mouse images were generated in situ on sagitally cut but unfixed mouse heads, but no differences between knockout and control littermates were observed. Absence of specific brain abnormalities in phenylalanine hydroxylasedeficient mice contrasts with the increased demyelination of periventricular deep cerebral white matter observed in human PKU patients.

Cranial MRI also enabled the detection of focal encephalitis after intranasal inoculation of healthy mice with herpes simplex virus. ${ }^{6}$ In analogy with human patients with encephalitis caused by herpes simplex virus, decreased signal intensity was observed on $\mathrm{T}_{1}$-weighted images in isolated foci throughout the mouse brain, while an increased signal intensity was observed on $\mathrm{T}_{2}$-weighted images. Subsequent histopathological findings on the same animals correlated well with the MRI findings.

A very elegant experimental design was used to study the end stage of scrapie. ${ }^{7}$ As mice infected with scrapie have a short life span, transgenic neural tissue overexpressing the normal cellular prior protein PrPc was transplanted into the forebrain of Prnp knockout mice and infected with scrapie prions. Because the knockout mice are refractory to scrapie, the disease remains restricted to the graft, and consequently the mice live much longer than scrapie-sensitive mice do. Using predominantly $\mathrm{T}_{1}$-weighted $\mathrm{MRI}$, this enabled the assessment of changes of this part of the brain over a prolonged period of time. Spongiosis with small vacuoles was encountered after 70-140 days of infection. Giant vacuolisation occurs after 150-300 days, but disappeared at later stages (450 days).

Construction of a mouse model for the neurodegenerative lysosomal storage disease aspartylglucosaminuria (AGU), by disrupting exon 8 of the aspartylglucosaminidase gene (AGA) allowed comparison of brain pathology between the human syndrome and its mouse model. ${ }^{8}$ In man, cerebral atrophy is visible as an increased volume of the lateral ventricles. In addition, deep gray matter structures are reported hypointense on MRI recordings. Homozygous AGA mice were recorded by $\mathrm{T}_{2}$-weighted MRI at the age of 6 months and shown to have prominent ventricles and hypointense deep gray matter structures as compared to cortical gray matter. The neuropathology of the mouse model thus closely resembles the human situation.

Two in vivo studies on the neuroanatomy of the mouse brain of two different knockout mouse models for human mental retardation syndromes have been performed by our group. In both studies, full 3D images of the whole mouse brain were generated. In addition to a descriptive study of the neuroanatomy of the mutant mouse models, we measured the size of the various brain structures in the mouse models reported abnormal in the corresponding human syndromes, thus using more of the potentials of MRI.
The first knockout mouse model studied by us was an L1CAM knockout. Mutations in the L1CAM gene on Xq28 in man cause a variety of neurological disorders, including X-linked hydrocephalus. ${ }^{9}$ Initial analysis suggested that the L1CAM knockout model was a good model for the human disease, as in parallel with human patients, L1CAM knockout mice showed errors in axon guidance at the corticospinal tract, ${ }^{10}$ and deficits in cognitive function tests. ${ }^{11}$ However, using conventional, pathological techniques, we were unable to detect hydrocephalus in the mouse model. Thus, we imaged the whole mouse brain and compared it with the control littermates. 3D reconstructions were made and the brain regions relevant in human pathology identified and delineated. Subsequently the volumes of the various ventricular systems and brain regions were calculated according to a newly defined algorithm. ${ }^{12}$ Only after careful examination of the images and statistical analysis of the data, were we able to demonstrate ventricular dilatation of the 4th and lateral, but not of the 3rd ventricle, elongation of the aqueduct of Sylvius, and cerebellar vermis hypoplasia (Figure 5). It has to be added though, that another group that independently constructed an L1CAM knockout mouse model ${ }^{13}$ did see large hydrocephalus when the knockout mice were bred in a $129 \times \mathrm{C}_{57} \mathrm{Bl} / 6$ background, while no abnormalities in ventricular size were noted when these, like our L1CAM knockouts, were bred in a pure 129 background.

Another mouse model imaged by us extensively is the FMR1 knockout mouse, an animal model for fragile $\mathrm{X}$ syndrome. This is the most common form of inherited

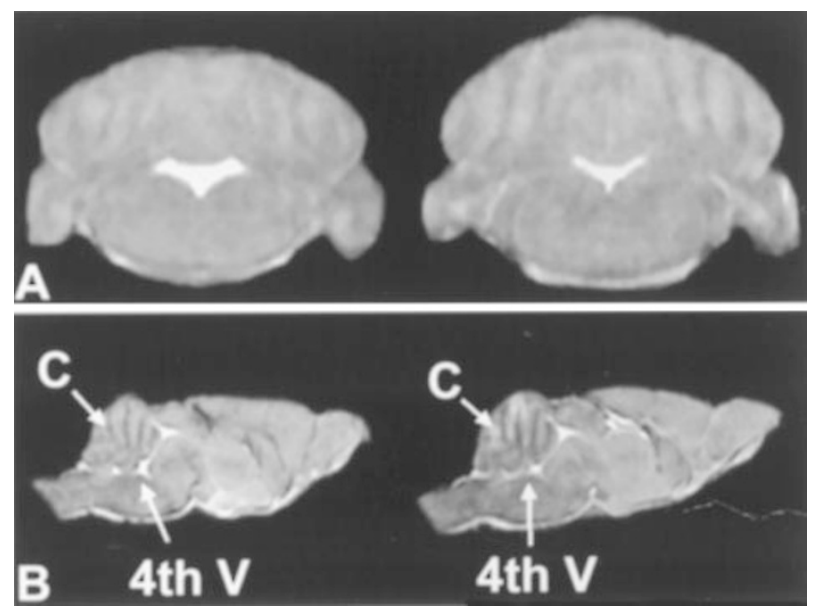

Figure 5 (A) Two in vivo obtained coronal images of the mouse brain, taken at the level of the 4 th ventricle, with on the left, the brain of an L1- knockout, showing a different sized brain and cerebellum and a differently shaped 4 th ventricle. As compared to the normal littermate on the right. (B) Two in vivo obtained midsagital images of the mouse brain, with an L1-knockout on the left, showing a vermis (C) with a smaller size and a different shape, as compared to the littermate on the right. 
mental retardation, characterised by mild to moderate mental retardation, mild dysmorphic features, macroorchidism (enlarged testicles) and behavioural problems, including hyperactivity and autistiform behaviour. ${ }^{14}$ The mouse model has been studied extensively, and shown to be a good model for the human disorder, exhibiting abnormalities in cognitive function tests and macroorchidism. ${ }^{15,16}$ MRI recordings of the mouse model were initiated because MRI studies in human patients reported several brain abnormalities, including a smaller size of the cerebellar vermis and ventricular dilatation, abnormalities that could potentially be linked to the behavioral problems of patients. However, no abnormalities were found in the brain structures of the knockout mice that were previously identified in humans. ${ }^{17}$

\section{Lessons from initial studies}

This overview illustrates that with current technology it is possible to image the mouse brain in vivo and inspect its major regions, including cerebellum, olfactory bulb, brain stem, hippocampus, cortical grey, etc. Longitudinal studies on one and the same animal have been performed, ${ }^{7}$ and volumetric measurements of different parts of the brain are feasible. ${ }^{11,17}$ In general, with the aid of a specific mouse brain atlas ${ }^{18}$ the various brain regions are readily identified as the MRI images resemble the histological sections. However, on MRI the size and shape of the various components of the ventricular system differ markedly from histological views. In particular, the lateral ventricles that appear as major components on fixed and stained sections are hardly visible on sagittal MRI recordings. This is due to their smaller shape, as illustrated on coronal MRI images. Apparently the ventricular system is severely affected by the fixation and/or staining procedures inevitable in pathological research.

The neuroanatomy of some of these mouse models corresponds well with the abnormalities described in the corresponding human disorders. For example, the mice infected with scrapie showed all the characteristics thought typical of the human infection and the AGA mice did show prominent ventricles and hypointense deep grey matter structures as observed in humans affected with this lysosomal storage disease. In other mouse models, the relation between human and mouse neuropathology is less obvious: The PKU knockout does not show evidence for increased demyelation of deep cerebral white mater thought characteristic for human patients; fragile $\mathrm{X}$ mice show no evidence for vermis hypoplasia or ventricular dilatation repeatedly reported in human patients; L1CAM mice show ventricular dilatation of lateral and 4th ventricle, reported abnormal in human patients, but not of the 3rd, which is also reported to be enlarged in human patients.

The authors of these studies acknowledge the differences between human and mouse pathology, but point out that human pathology is often neither completely nor correctly described, and raise several questions: Is increased demyelinisation of deep cerebral white matter characteristic for human PKU patients?; Do fragile X patients really have a brain structure different from controls?; What is the brain pathology of patients with mutations in the L1CAM gene? It is, for instance, not known whether the vermis hypoplasia and the increased size of the 4 th ventricle is a general feature of human L1CAM patients. The cerebellar structures of only five patients with this rare disease have been imaged, and although four of those appeared to have a smaller size of the midsagittal cerebellar structure, the small number of patients analysed in this study makes it impossible to draw general conclusions regarding the size of the cerebellar vermis in human patients.

Another explanation for the observed differences in pathology between human patients and the corresponding knockout mice may be the influence of genetic background. It is well established that genetic background may have a profound influence on the various genetic manipulations in mice. ${ }^{19}$ For example, deletion of the hypoxanthine phosphoribosyl-transferase (HPRT) results in Lesch-Nyhan disease in man but has no apparent effect in mice. ${ }^{20}$ Some patients with X-linked hydrocephalus show major enlargement of the ventricular system (Figure 1), while other members of the

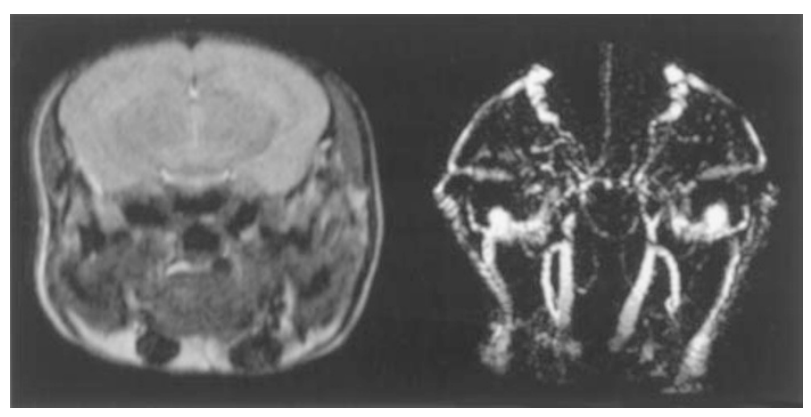

Figure 6 In vivo obtained coronal MR image of the head of a mouse on the left, and maximal signal intensity projections in the coronal plane of the corresponding blood vessels (angiography).

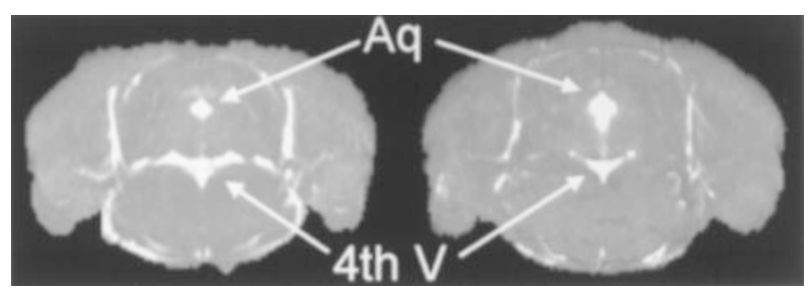

Figure 7 Maximal signal intensity projections in the coronal plane of the cerebrospinal fluid filled spaces in the brain of an L1knockout mouse on the left and of a control littermate on the right displaying the difference in size and shape of the 4th ventricle and the aqueduct (ventriculography). 
same family may show only a very limited enlargement as a consequence of the same L1CAM mutation. ${ }^{9}$ This situation is reflected in the knockout mouse model, where gross hydrocephalus is observed when the mice are bred in a $129 \times \mathrm{C}_{57} \mathrm{Bl} / 6$ mixed genetic background, while only a small enlargement of the ventricular system is observed when the mice are bred in a pure 129 background. ${ }^{11,13}$

\section{Perspective}

It has been demonstrated that detailed mouse brain imaging providing high resolution morphological information from a living mouse is feasible using MRI microscopy with current technology. Nonetheless, the resolution of the technique may still increase with the development of new MRI equipment, new software for image analysis and with improved pulse-sequences to induce magnetic resonance.

Perhaps even more exciting MRI developments include spin-off techniques which share the characteristic that the signal is derived from proton resonance. However, the contrast is no longer a function of proton density, $\mathrm{T}_{1}$ and $\mathrm{T}_{2}$ only, but also from physiological parameters. For instance, functional magnetic resonance imaging (fMRI) allows the study of regional activity in the living brain. The contrast in these images is based on changes in the local blood perfusion and/or increase in local oxyhaemoglobin $v s$ deoxyhaemoglobin ratio. ${ }^{21,22}$ and is then referred to as Blood Oxygenation Level Dependent (BOLD) contrast. Using BOLD MRI (fMRI) it has been feasible to trace brain activation in rats $^{23}$ and initial results in mice are promising. ${ }^{24}$ In addition, the MRI contrast can also be used to measure flow rates in blood vessels or to obtain high resolution images of the vascular bed or cerebrospinal fluid filled spaces in the case of angiography ${ }^{25}$ (Figure 6) and ventriculography (Figure 7), respectively.

A major potential of MRI is that different measurements can be combined in a single examination. This will offer the possibility of comprehensive brain studies, combining anatomical with functional information. Such an integrated picture of the brain may help understand brain (mal)function in genetically modified mouse models.

\section{Acknowledgements}

Financial support for the research performed in Antwerp was obtained through grants from the University of Antwerp, the Belgian National Fund for Scientific Research - Flanders (FWO), the Fragile $X$ research foundation (FRAXA) and from the EC. Research in Vance Lemmon's laboratory is supported by the NEI and NINDS.

\section{References}

1 Martin JE, Fisher EMC: Phenotypic analysis - making the most of your mouse. Trends Genet 1997; 13: 254-256.

2 Boesch C: Molecular aspects off magnetic resonance imaging and spectroscopy. Mol Aspects Med 1999; 20: 185-318.
3 Russo E: Going micro: imaging devices to benefit both mouse and biologist. Scientist 1998; 12: http://www.the-scientist.com/ yr1998/oct/russo_p1_981026.html

4 Kornguth S, Bersu E, Anderson M, Markley J: Correlation of increased levels of class $1 \mathrm{MHC} \mathrm{H}-2 \mathrm{Kk}$ in the placenta of murine trisomy 16 conceptuses with structural abnormalities revealed by magnetic resonance microscopy. Teratology 1992; 45: 383 391.

5 Kornguth S, Anderson M, Markley JL, Shedlovsky A: Nearmicroscopic magnetic resonance imaging of the brains of phenylalanine hydroxylase-deficient mice, normal littermates, and of normal BALB/c mice at 9.4 Tesla. Neuroimage 1994; 1 : $220-229$

6 Lamadé UM, Lamadé W, Hess T et al: A mouse model of herpes simplex virus encephalitis: diagnostic brain imaging by magnetic resonance imaging. In Vivo 1996; 10: 563-568.

7 Brandner S, Isenmann S, Kühne G, Aguzzi A: Identification of the end stage of scrapie using infected neural grafts. Brain Pathol 1998; 8: 19-27.

8 Jalanko A, Tenhunen K, McKinney CE et al: Mice with an aspartylglucosaminuria mutation similar to humans replicate the pathophysiology in patients. Hum Mol Genet 1998; 7: 265 272.

9 Fransen E, Van Camp G, Vits L, Willems PJ: L1-associated diseases: clinical geneticists divide, molecular geneticists unite. Hum Mol Genet 1997; 6: 1625 - 1632.

10 Cohen NR, Taylor JSH, Scott LB, Guillery RW, Soriano P, Furley AJW: Errors in corticospinal axon guidance in mice lacking the neuronal cell adhesion molecule L1. Curr Biol 1997; 8: 26-33.

11 Fransen E, D'Hooge R, Van Camp G et al: L1 knockout mice show dilated ventricles, vermis hypoplasia and impaired exploration patterns. Hum Mol Genet 1998; 7: 999-1009.

12 Sijbers J, Scheunders P, Verhoye M, Van der Linden A, Van Dyck D, Raman E: Watershed-based segmentation of 3D MR data for volume quantization. Magn Reson Imaging 1997; 15: 679-688.

13 Dahme M, Bartsch U, Martini R, Anliker B, Schachner M, Mantei $\mathrm{N}$ : Disruption of the mouse L1 gene leads to malformations of the nervous system. Nat Genet 1997; 17: 346-349.

14 Kooy RF, Willemsen R, Oostra BA: Fragile X syndrome at the turn of the century. Mol Med Today 2000; 6: 194-199.

15 Bakker CE, Verheij C, Willemsen R et al: Fmr1 knockout mice: a model to study fragile X mental retardation. Cell 1994; 78: 23 33.

16 Kooy RF, D'Hooge R, Reyniers E et al: Transgenic mouse model for the fragile X syndrome. Am J Med Genet 1996; 64: 241-245.

17 Kooy RF, Reyniers E, Verhoye $M$ et al: Neuroanatomy of the fragile $\mathrm{X}$ knockout mouse brain studied using in vivo high resolution magnetic resonance imaging (MRI). Eur J Hum Genet 1999; 7: 526-532.

18 Franklin KBJ, Paxinos G: The mouse brain in stereotaxic coordinates. San Diego, CA, Academic Press, 1997.

19 Livy DJ, Wahlsten D: Tests of genetic allelism between four inbred mouse strains with absent corpus callosum. J Hered 1991; 82: $459-464$.

20 Hooper M, Hardy K, Handyside A, Hunter S, Monk M: HPRTdeficient (Lesch-Nyhan) mouse embryos derived from germline colonization by cultured cells. Nature 1987; 326: 292-295.

21 Belliveau JW, Kennedy DNJ, McKinstry RC et al: Functional mapping of the human visual cortex by magnetic resonance imaging. Science 1991; 254: 716-719.

22 Ogawa S, Tank DW, Menon R et al: Intrinsic signal changes accompanying sensory stimulation: functional brain mapping with magnetic resonance imaging. Proc Natl Acad Sci USA 1992; 89: $5951-5955$

23 Peeters RR, Verhoye M, Vos BP, Van Dyck D, Van der Linden A, De Schutter E: A patchy horizontal organization of somatosensory activation of the rat cerebellum demonstrated by functional MRI. Eur J Neurosci 1999; 11: 2720 - 2730. 
24 Huang W, Plyka I, Li H, Eisenstein EM, Volkow ND, Springer CSJ: Magnetic resonance imaging (MRI) detection of the murine brain response to light: temporal differentiation and negative functional MRI changes. Proc Natl Acad Sci USA 1996; 93: 6037 6042 .
25 Beckmann N, Stirnimann R, Bochelen D: High-resolution magnetic resonance angiography of the mouse brain: application to murine focal cerebral ischemia models. J Magn Reson 1999; 140: $442-450$. 